\title{
Diagnóstico situacional dos serviços ambulatoriais de reabilitação física do SUS em Salvador-Ba
}

\author{
Situational diagnosis of physical rehabilitation services in the SUS of Salvador-Ba
}

\begin{abstract}
Fabiane Costa Santos ${ }^{1}$, Ediná Alves Costa², Italo Ricardo Santos Aleluia ${ }^{3 *}$, Elzo Pereira Pinto Júnior*
Mestre em Saúde Comunitária pelo Instituto de Saúde Coletiva (IC/UFBA). Fisioterapeuta da Secretaria Estadual de Saúde da Bahia (SESAB).; ${ }^{2}$ Doutora em Saúde Pública pela Universidade de São Paulo, Professora associada do Instituto de Saúde Coletiva da Universidade Federal da Bahia (ISC/UFBA).; 3 Mestre em Saúde Coletiva (ISC) UFBA) e Doutorando em Saúde Pública, Professor Assistente da Universidade Federal do Oeste da Bahia (UFOB). ${ }^{4}$ Mestre em Saúde Coletiva pelo Programa de Pós-Graduação em Saúde Coletiva da Universidade Estadual do Ceará, Doutorando em Saúde Pública pelo Programa de Pós-Graduação em Saúde Coletiva do ISC-UFBA.
\end{abstract}

\begin{abstract}
Resumo
Objetivo: realizar um diagnóstico da situação dos serviços ambulatoriais de reabilitação física do SUS na cidade de Salvador, Bahia. Metodologia: a análise diagnóstica foi realizada em 12 serviços, a partir de entrevistas semiestruturadas; observação participante; consulta a dados do cadastro dos estabelecimentos e dos planos de saúde. A análise dos dados considerou elementos teóricos do planejamento estratégico-situacional à luz de "uma árvore de problemas". Resultados: insuficiência na infraestrutura dos serviços (sobretudo de profissionais); ausência de serviços da rede própria, com influência direta sobre a organização da agenda e estabelecimento de fluxos assistenciais; barreiras geográficas ao acesso; ausência de estratégias de educação permanente; considerável demanda reprimida; problemas de repasses e suficiência de recursos financeiros; e importantes divergências entre dados cadastrais dos sistemas de informação e a realidade encontrada, foram os principais problemas evidentes. Conclusão: o presente diagnóstico evidenciou entraves importantes à garantia do direito à saúde em ações de reabilitação física, que transitaram da dimensão infraestrutural à político-institucional. Os resultados aqui evidenciados permitiram identificar a necessidade de análises políticas e estratégicas envolvendo a assistência em reabilitação em saúde.

Palavras-chave: Serviços de Reabilitação. Assistência à Saúde. Administração de Serviços de Saúde. Sistema Único de Saúde.
\end{abstract}

\begin{abstract}
Objective: to perform a diagnosis of the outpatient physical rehabilitation services of SUS in the city of Salvador, Bahia. Methodology: the diagnostic analysis was performed in 12 services, based on semi-structured interviews; participant observation; data from the register of establishments and health plans. Data analysis considered theoretical elements of strategic-situational planning in the light of "a tree of problems". Results: insufficiency in the infrastructure of services (especially professionals); lack of services of the own network, with direct influence on the organization of the agenda and establishment of assistance flows; geographical barriers to access; absence of permanent education strategies; considerable pent-up demand; problems of onlending and sufficiency of financial resources; and important divergences between cadastral data of the information systems and the reality found, were the main evident problems. Conclusion: the present diagnosis evidenced important obstacles to the guarantee of the right to health in actions of physical rehabilitation, that transited from the infrastructural dimension to the political-institutional one. The results showed that the need for political and strategic analysis involving health rehabilitation assistance was identified.

Keywords: Rehabilitation Services. Delivery of Health Care. Health Services Administration. Unified Health System.
\end{abstract}

\section{INTRODUÇÃO}

O Relatório Mundial sobre Deficiência aponta que mais de um bilhão de pessoas são portadores dessa condição e que cerca de 200 milhões apresentam dificuldades funcionais significativas para a realização das suas atividades de vida diária (OMS, 2011). No Brasil, desde 2002 já se estimava a existência de 18 milhões de pessoas com deficiência ( $P c D)$, cujas principais causas estão relacionadas aos transtornos congênitos, doenças

Correspondente/Corresponding: *Italo Ricardo Santos Aleluia - End: Estrada do Barrocão, s/n, Prainha, Barreiras, Bahia, Brasil. - Tel: (75) 99128-2395. -E-mail: italoaleluia@ufob.edu.br transmissíveis e crônicas não transmissíveis, perturbações psiquiátricas, abuso de álcool, drogas e desnutrição (BRASIL, 2011). Além dessas causas, cabe destacar o aumento nos indicadores de morbidade em decorrência dos acidentes e violências, que conferem às vítimas algum grau de incapacidade funciona (REICHENNHEIM et al., 2011), e a ampliação da expectativa de vida, que contribuiu para ocorrência de doenças crônico-degenerativas em idosos (SCHMIDT et al., 2011).

Com o crescimento das incapacidades funcionais emerge o desafio para o SUS de garantir atenção integral à saúde das pessoas com deficiência, sobretudo no âmbito da reabilitação. Isso remonta para estruturação ideal dos serviços de saúde, que seja capaz de atender as 
principais necessidades dos sujeitos, de modo reinseri-los na sociedade (BONARD et al., 2007).

A incipiente organização da assistência em reabilitação ainda é evidente em diferentes regiões no mundo. Na América Latina e no Caribe, os poucos serviços de reabilitação existentes apresentavam baixa qualidade e os objetivos do tratamento raramente eram cumpridos, pois a assistência em reabilitação não representava uma prioridade no setor público (OPAS, 1996).

No Brasil, a fragilidade da assistência em reabilitação é prévia à institucionalização do Sistema Único de Saúde (SUS). As primeiras instituições brasileiras criadas para atender à demanda da reabilitação surgiram na década de 40 , ainda assim, restritas às entidades filantrópicas. Vinte anos depois, as únicas instituições públicas responsáveis pelas ações de reabilitação eram os centros de reabilitação profissional do Instituto Nacional de Previdência Social (INPS) e não haviam políticas públicas destinadas para este fim (RIBEIRO et al., 2010).

A institucionalização do SUS criou um cenário mais propício à implantação de programas de reabilitação. Entretanto, isso não significou maior organização dos serviços de reabilitação, em virtude da precária estruturação da rede assistencial, que nunca contemplou uma política integral de atendimento às incapacidades (BRASIL, 2002).

Surgiram também as primeiras iniciativas normativas do Ministério da Saúde (MS) no sentido de estabelecer ações diretivas às PcD. Dentre elas, cabe destacar a Política Nacional de Saúde da Pessoa com Deficiência, criada em 2002, com o objetivo de propiciar atenção integral à saúde, incluindo a concessão de órteses, próteses e meios auxiliares de locomoção, quando necessários (KIELMANN; SILVEIRA; SOUZA, 2011). Além disso, importante arcabouço legal buscou normatizar a organização da assistência de reabilitação no SUS relacionada a serviços e procedimentos; organização e implantação de redes estaduais de atenção e financiamento (ALMEIDA; CAMPOS, 2002; CAVALCANTI, 2009; LIMA et al., 2009).

A mais recente e importante iniciativa do poder público incluiu na agenda do Estado os cuidados à pessoa com deficiência no âmbito do SUS, com a oferta de serviços de reabilitação integrados, articulados e efetivos nos diferentes pontos de atenção (BRASIL, 2010). Ademais, as políticas de Atenção à Saúde do Idoso e de Redução de Morbimortalidade por Acidentes e Violências (PNRMAV), também preveem a necessidade da consolidação do atendimento em reabilitação no SUS, inclusive com garantia de infraestrutura dos serviços, de modo que esses passaram a assumir certa transversalidade no sistema de saúde brasileiro (CAVALCANTI, 2009; DESLANDES et al., 2006).

Apesar do avanço na criação de amplo arcabouço legal, persistem ações fragmentadas e desvinculadas do processo de reabilitação (Prefeitura Municipal de Salvador, 2010), além do número reduzido de unidades de reabilitação disponíveis no país, concentrados na iniciativa privada e com características centralizadoras (LIMA et al., 2009).

Evidências apontam uma série de problemas que atingem os serviços de média complexidade, onde também se inserem os serviços de reabilitação. São comuns dificuldade de acesso e baixo grau de integração com outros níveis de atenção; falta de planejamento das ações e da articulação com o perfil epidemiológico da população; subfinanciamento para qualificação profissional e baixa regulação da oferta (TEIXEIRA, 2010).

Os achados aqui delineados convergem com evidências que ratificam a organização dos serviços de reabilitação como eixo crítico no sistema de saúde brasileiro, demandando urgente e contínuo aporte financeiro substancial do governo federal (LIMA et al., 2009). A identificação das falhas dos serviços disponíveis pode representar um ponto de partida para o planejamento das ações a serem implementadas.

O levantamento da literatura sobre a temática revelou importantes lacunas existentes no conhecimento sobre a situação de Salvador-Ba, maior capital do Nordeste e contexto de profundas desigualdades sociais, situada em tipologia regional de baixo desenvolvimento socioeconômico e baixa oferta de serviços de saúde (CONSENSUS, 2011). Considerando a magnitude do problema e a importância de um adequado funcionamento destes serviços para atendimento das necessidades dos usuários, o presente estudo teve como objetivo realizar um diagnóstico da situação dos serviços ambulatoriais de reabilitação física do SUS na cidade de Salvador, Bahia.

\section{METODOLOGIA}

Trata-se de um estudo avaliativo com abordagem descritiva e qualitativa. $O$ estudo foi realizado de forma extensiva em 12 serviços de reabilitação e teve dois níveis de análise: os serviços e a gestão.

Os serviços foram selecionados considerando os seguintes critérios: possuir registro no Cadastro Nacional dos Estabelecimentos de Saúde (CNES) denominado como serviço de reabilitação; e ser vinculado ao Sistema Único de Saúde, direta ou indiretamente. A seleção foi realizada, inicialmente, a partir da consulta ao CNES e refinada com informações consultadas nos sítios eletrônicos de cada serviço (quando possível). Ao final desse processo, foram pré-selecionados 12 serviços para realização do diagnóstico situacional.

A produção dos dados combinou a utilização de fontes secundárias e primárias. Sobre as primeiras utilizou-se o CNES, o Plano Estadual de Saúde da Bahia (2007-2010) e o Plano Municipal de Saúde (PMS) de Salvador (2010-2013). Sobre as segundas foram realizadas entrevistas semiestruturadas com técnicos do nível central, gerentes dos serviços, além da observação participante.

Inicialmente realizou-se a consulta aos planos de saúde supracitados com o objetivo de identificar as ações planejadas para os serviços e de orientar a elaboração dos roteiros de entrevista.

Em seguida, consultou-se os dados do CNES para 
caracterização dos serviços, a partir de informações relativas à infraestrutura dos serviços (instalações físicas para assistência ambulatorial, equipamentos disponíveis e quadro de profissionais para desenvolvimento das atividades); elementos da organização e gestão dos serviços (tipo de unidade, esfera administrativa, natureza da organização, tipo de gestão e existência de contrato de gestão e metas) e características da prestação de serviços (serviços especializados oferecidos, serviços de apoio, fluxo de clientela).

Para seleção dos entrevistados contou-se com o apoio de informantes-chave das secretarias municipal e estadual de saúde e adotou-se os seguintes critérios de inclusão: participarem da gerência dos serviços selecionados há pelo menos um ano, para os técnicos do nível central da SESAB e SMS. Para o âmbito estadual os informantes deveriam compor a diretoria de planejamento, a Superintendência de Atenção Integral à Saúde (SAIS) e a Diretoria de Gestão do Cuidado (DGC); e no âmbito municipal, a Coordenadoria de Atenção e Promoção à Saúde (COAPS) e a Coordenaria de Regulação e Avaliação
(CRA). Essas áreas foram escolhidas estrategicamente, por se tratarem de espaços diretamente relacionados à gestão da assistência em reabilitação.

Nas entrevistas com os técnicos do nível central questionou-se sobre as características do planejamento dos serviços e dos parâmetros e normas estabelecidos para seu funcionamento. Já com os gerentes investigou-se sobre a organização assistencial dos serviços; planejamento e parâmetros estabelecidos para funcionamento; principais problemas enfrentados; fluxos assistenciais; capacidade instalada; lista de espera; e atividades realizadas conforme o estabelecido nas disposições legais sobre reabilitação física em saúde.

Apesar de pré-selecionados 12 serviços (Quadro 1), apenas foi possível a realização de entrevistas com gerentes de sete deles (serviço 1 a 7), tanto pelas dificuldades de acesso aos gerentes, como a um serviço, que existia no cadastro, mas não na prática (foi extinto). Desta forma, esse estudo contou com um total de 11 serviços investigados. Todas as entrevistas foram gravadas, transcritas na íntegra e revisadas pelos pesquisadores.

Quadro 1 - Serviços de reabilitação investigados.

\begin{tabular}{|c|c|c|c|}
\hline Serviço & Cadastros no CNES & & Cadastros no CNES \\
\hline 1 & $\begin{array}{l}\text { Serviço de Reabilitação Física } \\
\text { Serviço de Reabilitação Intelectual }\end{array}$ & 7 & $\begin{array}{l}\text { Serviço de Reabilitação Física } \\
\text { Serviço de Reabilitação Intelectual } \\
\text { Serviço de Reabilitação para Múltiplas Deficiências }\end{array}$ \\
\hline 2 & $\begin{array}{l}\text { Serviço de Reabilitação Física } \\
\text { Serviço de Fisioterapia }\end{array}$ & 8 & $\begin{array}{l}\text { Serviço de Reabilitação Física } \\
\text { Serviço de Fisioterapia } \\
\text { Serviço de Reabilitação Intelectual } \\
\text { Serviço de Reabilitação para Múltiplas Deficiências }\end{array}$ \\
\hline 3 & $\begin{array}{l}\text { Serviço de Reabilitação Física } \\
\text { Serviço de Fisioterapia }\end{array}$ & 9 & $\begin{array}{l}\text { Serviço de Reabilitação Física } \\
\text { Serviço de Fisioterapia } \\
\text { Serviço de Reabilitação Intelectual } \\
\text { Serviço de Reabilitação para Múltiplas Deficiências }\end{array}$ \\
\hline 5 & $\begin{array}{l}\text { Serviço de Reabilitação Física } \\
\text { Serviço de Fisioterapia }\end{array}$ & 11 & $\begin{array}{l}\text { Serviço de Reabilitação Física } \\
\text { Serviço de Fisioterapia } \\
\text { Serviço de Reabilitação Intelectual } \\
\text { Serviço de Reabilitação para Múltiplas Deficiências }\end{array}$ \\
\hline 6 & $\begin{array}{l}\text { Serviço de Reabilitação Física } \\
\text { Serviço de Fisioterapia }\end{array}$ & 12 & Sem informação \\
\hline
\end{tabular}

Fonte: Elaboração dos autores. Adaptado por Cadastro Nacional dos Estabelecimentos de Saúde (CNES).

A observação participante foi também utilizada como técnica de produção dos dados e nela foram observados e registrados em diário de campo, fatos relevantes e situações vivenciadas nas visitas aos serviços (incluindo aqueles em que seus gerentes não foram entrevistados) e nas visitas em áreas estratégicas do nível central da SMS e SESAB.
As informações provenientes das entrevistas, dados secundários e do diário de campo foram trianguladas para compor uma matriz de análise à luz das dimensões sumarizadas no Quadro 2. 
Quadro 2 - Sumarização das dimensões, categorias operacionais e fontes de evidência.

\begin{tabular}{|l|l|l|}
\hline Dimensão & Categorias Operacionais & Fonte \\
\hline Infraestrutura & $\begin{array}{l}\text { Instalações físicas para assistência ambulatorial; equipamentos disponíveis } \\
\text { para reabilitação física e quadro de profissionais para desenvolvimento das } \\
\text { atividades. }\end{array}$ & $\begin{array}{l}\text { CNES, observação participante e entre- } \\
\text { vistas com gerentes. }\end{array}$ \\
\hline Financiamento & $\begin{array}{l}\text { Suficiência dos recursos; formas de repasse; investimentos nos serviços e na } \\
\text { rede de reabilitação. }\end{array}$ & Entrevistas com técnicos e gerentes. \\
\hline Organização e gestão & $\begin{array}{l}\text { Tipo de unidade; esfera administrativa; natureza da organização; tipo de gestão; } \\
\text { existência de contrato de gestão e metas; parâmetros e normas de funciona- observação participante e planos } \\
\text { mento e planejamento de ações para os serviços. }\end{array}$ & $\begin{array}{l}\text { CNÉ de. } \\
\text { de saúde. }\end{array}$ \\
\hline Prestação & $\begin{array}{l}\text { Serviços especializados oferecidos; serviços de apoio; fluxo de clientela; capa- } \\
\text { cidade instalada; tempo de espera para o primeiro atendimento e atividades } \\
\text { realizadas. }\end{array}$ & Entrevistas com técnicos e gerentes. \\
\hline
\end{tabular}

Fonte: Elaboração dos autores. Adaptado por Cadastro Nacional dos Estabelecimentos de Saúde (CNES).

Para as categorias que exigiram a verificação normativa de atendimento aos parâmetros estabelecidos, foram utilizados como referência os parâmetros de funcionamento para serviços de reabilitação física dispostos na Portaria MS/GM nº 818 (DESLANDES et al., 2006).

Os serviços foram analisados de forma comparativa e para isso, triangulou-se os dados e as fontes de evidência em uma matriz, segundo as dimensões e categorias operacionais, por serviço. Em seguida, foram construídos argumentos a partir das evidências e estes foram cotejados com os elementos teóricos do momento explicativo Planejamento Estratégico Situacional (PES), a saber: o problema, a situação e o diagnóstico administrativo (TESTA, 1995).

Para esse estudo considerou-se como momento explicativo o levantamento da situação inicial, pressupondo a identificação, descrição e análise dos problemas (algo considerado fora dos padrões de normalidade para um ator social e que gera cara negativa), a fim de entender a situação (como um conjunto de problemas e/ou necessidades tal como são compreendidos a partir da perspectiva dos atores sociais interessados em intervir, sobre um determinado recorte da realidade), a partir do diagnóstico administrativo (identificação dos recursos, atividades e nós técnicos críticos) (TESTA, 1995).

A partir da categorização dos resultados construiu-se uma explicação dos problemas diagnosticados nos serviços, tomando como referência a construção de uma "árvore dos problemas" e categorias teóricas supracitadas, as quais serão apresentadas na seção de resultados (LIMA, 2012; TESTA, 1995).

O projeto dessa pesquisa foi aprovado pelo Comitê de Ética em Pesquisa (CEP) do Instituto de Saúde Coletiva (ISC), da Universidade Federal da Bahia (UFBA), sob parecer no 030/12. Todos os indivíduos entrevistados assinaram um Termo de Consentimento Livre e Esclarecido e foi garantido o anonimato dos entrevistados e das instituições na apresentação dos resultados. Os serviços participantes da pesquisa concederam anuência institucional aos pesquisadores.

\section{RESULTADOS}

A seguir, será apresentada a caracterização geral dos serviços investigados (Tabela 1) e posteriormente será explicitada a respectiva situação, a partir dos problemas encontrados segundo as dimensões de análise já descritas (Tabela 2). Por questões éticas, os serviços serão representados por números.

Quando analisadas suas características e requisitos normativos do MS, apenas três dos serviços investigados se enquadravam como de reabilitação física e o tipo de unidade mais frequente era ambulatório de especialidades. Evidenciou-se que a maioria dos serviços integra a iniciativa privada e verificou-se a inexistência de estabelecimentos pertencentes à rede própria municipal.

Tabela 1 - Caracterização sumarizada dos serviços de reabilitação física do SUS, Salvador, Bahia.

\begin{tabular}{|c|c|c|c|c|c|c|c|c|c|c|c|}
\hline \multirow{2}{*}{ Unidade de observação } & \multicolumn{11}{|c|}{ Serviço } \\
\hline & 1 & 2 & 3 & 4 & 5 & 6 & 7 & 8 & 9 & 10 & 11 \\
\hline \multicolumn{12}{|l|}{ Tipo de unidade } \\
\hline Ambulatório de especialidade & $\bullet$ & & $\bullet$ & & & & $\bullet$ & & & $\bullet$ & \\
\hline Ambulatório de Especialidade inserido em hospital geral & & & & & $\bullet$ & $\bullet$ & & & & & \\
\hline Centro de especialidade & & $\bullet$ & & & & & & & & & \\
\hline Clínica de especialidade & & & & $\bullet$ & & & & & $\bullet$ & & \\
\hline Hospital Especializado & & & & & & & & $\bullet$ & & & \\
\hline Policlínica & & & & & & & & & & & $\bullet$ \\
\hline Estadual & $\bullet$ & & $\bullet$ & & & & & & & & \\
\hline Federal & & & & & & - & & & & & \\
\hline
\end{tabular}




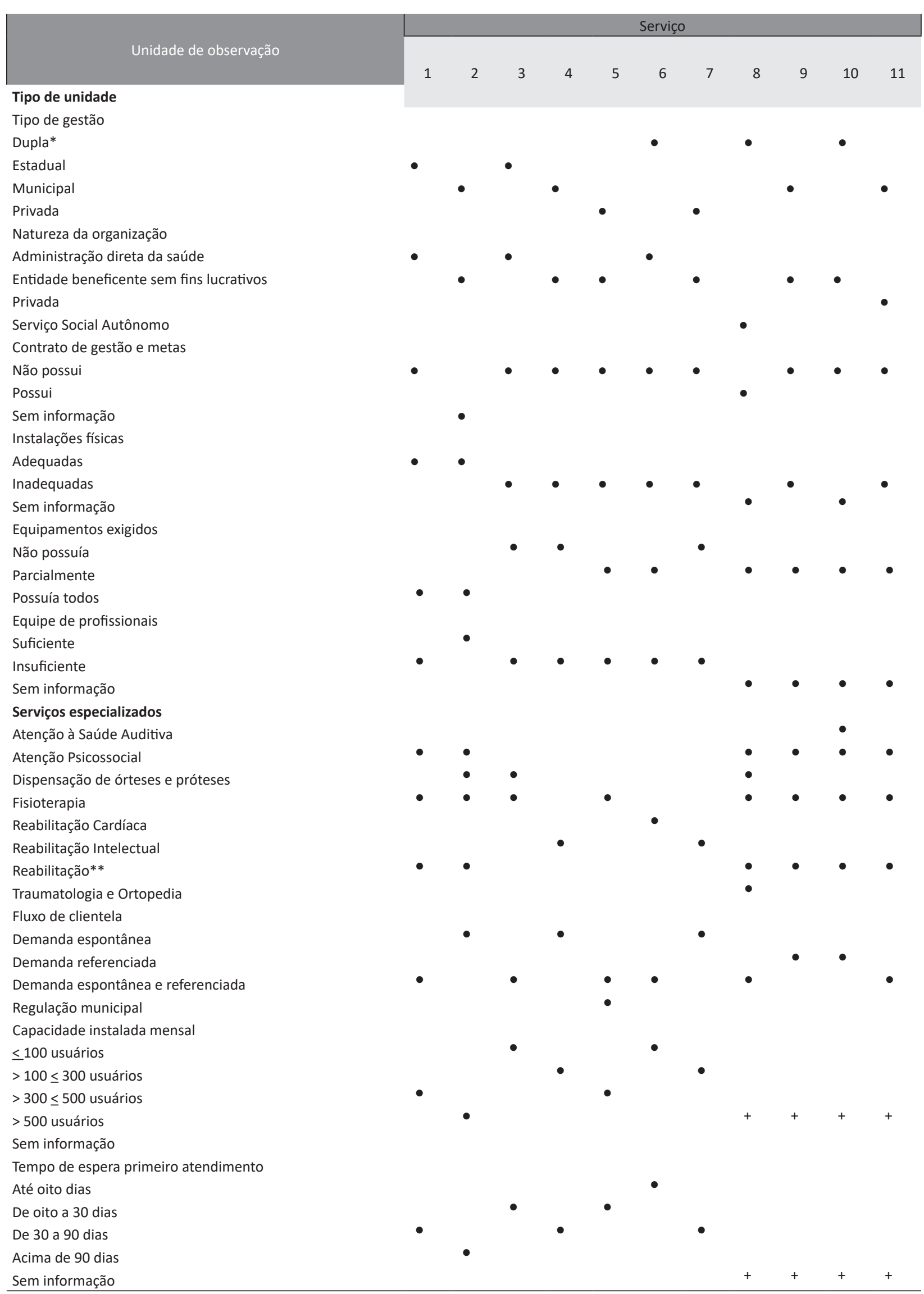

Fonte: Cadastro Nacional dos Estabelecimentos de Saúde (CNES) e entrevistas semiestruturadas.

Nota: + Sem informação; *Serviço gerido pelo setor público e privado simultaneamente; **Serviço de reabilitação multiprofissional. 
A gestão dos serviços era concentrada predominantemente na esfera privada, com pouquíssimos estabelecimentos administrados pelo município e estado, somada à uma importante insuficiência gerencial, dada ausência quase unânime de contratos de gestão e metas.

A maioria dos serviços possuía instalações físicas inadequadas para prestação das ações cadastradas e atendiam parcialmente à necessidade de equipamentos mínimos exigidos em portarias ministeriais. Havia insuficiência de profissionais em metade dos serviços analisados.

Os serviços especializados frequentemente ofertados correspondiam à atenção psicossocial, fisioterapia e reabilitação multiprofissional, cujo fluxo de clientela mais comum era a demanda espontânea e referenciada. Chamou a atenção uma importante pulverização da capacidade instalada mensal entre os serviços e o tempo estimado de espera para primeiro atendimento entre 30 a 90 dias.

Ao analisar a frequência dos problemas encontrados nos serviços, observou-se que a maior parte deles esteve circunscrito à infraestrutura e à organização e gestão. Em menor frequência haviam problemas ligados ao financiamento e prestação dos serviços.

Tabela 2 - Frequência de problemas dos serviços ambulatoriais de reabilitação física da cidade de Salvador, Bahia, segundo categorias.

\begin{tabular}{l|c|c|c|c|c|c|c}
\hline Problemas & Serviço 1 & Serviço 2 & Serviço 3 & Serviço 4 & Serviço 5 & Serviço 6 & Serviço 7 \\
\hline Infraestrutura & $\bullet$ & 0 & $\bullet$ & $\bullet$ & $\bullet$ & 0 & $\bullet$ \\
\hline Financiamento & 0 & 0 & 0 & $\bullet$ & 0 & 0 & $\bullet$ \\
\hline Gestão & $\bullet$ & $\bullet$ & $\bullet$ & 0 & $\bullet$ & $\bullet$ & 0 \\
\hline Prestação de serviços & 0 & 0 & $\bullet$ & 0 & $\bullet$ & 0 & $\bullet$ \\
\hline
\end{tabular}

Fonte: entrevistas semiestruturadas. Elaboração dos autores.

Nota: Existência de problemas 0 Ausência de problemas

Foi evidenciada uma série de incompatibilidades estruturais entre as informações do CNES com aquelas evidentes na observação e entrevistas com os gestores, no que tange às instalações físicas, profissionais, equipamentos e atividades realizadas. Apenas dois dos serviços visitados (1 e 2) possuíam infraestrutura adequada para um serviço de reabilitação física. Três deles (3, 4 e 7), embora cadastrados como serviços de reabilitação física, não dispunham de nenhum tipo de equipamento nas unidades para assistência aos pacientes. Em dois deles (3 e 6) o espaço físico era mínimo e comprometia a prestação do cuidado.

Foi referido descaso da gestão estadual para com os serviços que não estavam em funcionamento no Estado, o que acarretava numa insuficiência de funcionamento da rede estadual de reabilitação e trazia constrangimentos estruturais e organizacionais para aqueles serviços que funcionavam, a exemplo da alta demanda de usuários, com grande lista de espera para atendimento.

Diversos foram outros problemas evidenciados na infraestrutura. Dentre eles, destacaram-se a escassez de especialista em fisiatria no mercado; a falta de cadastro de diversos equipamentos encontrados na observação; serviços com incompatibilidade entre o cadastro e atividades realizadas; estrutura insuficiente, pelo inadequado espaço físico, falta e manutenção de equipamentos mínimos de reabilitação física; e número insuficiente de profissionais (evidente em cinco serviços).
O déficit de profissionais, associado à precária instalação física em um dos serviços reduziam as ações ao trabalho artesanal de profissional técnico-administrativo, que atuava em desvio de função como ortoprotesista. Essa precariedade não somente derivava de dificuldades de financiamento e critérios utilizados pela gestão para aquisição de equipamentos e contratação de pessoal, mas tinham relação com o desinteresse da direção de alguns estabelecimentos em investir, por exemplo, nos serviços de fisioterapia.

Havia problemas de acessibilidade geográfica em dois serviços, em virtude de sua localização periférica na capital. Isso prejudicava a assiduidade dos usuários e comprometia o acompanhamento dos casos em que era preciso monitoramento regular mediante novas avaliações ou teste de próteses, por exemplo.

Em relação ao financiamento, duas unidades referiram escassez de recursos financeiros. O repasse desses recursos em forma de blocos foi apontado como dificultador para priorização dos serviços de reabilitação física no momento do planejamento orçamentário.

Os problemas de organização e gestão evidenciados abarcavam a baixa remuneração dos profissionais; pouca liberdade das equipes para organizarem a agenda de trabalho, dada dificuldade de controle do quantitativo de pacientes em atendidos; ausência de investimentos na oficina ortopédica; além da inexistência de referência 
e contrarreferência nas unidades, pelo descumprimento dos protocolos assistenciais e ausência de comunicação entre os serviços.

A inexistência de serviços de reabilitação física próprios da rede municipal também foi um problema evidente, que atrelado ao mau funcionamento da rede estadual, sobrecarregava os serviços que apresentavam boa estrutura, de modo a não atender adequadamente a demanda.

A falta de capacitação profissional, a quantidade elevada de pacientes, a desmotivação para o trabalho e a falta de valorização profissional também foram relatos encontrados. Havia dificuldade para obtenção de dados epidemiológicos sobre deficiência no Estado e ausência de um espaço específico nos sistemas de informação, destinado a registro de dados sobre pessoas com deficiências, o que dificultava o dimensionamento da rede de serviços.

No que concerne à prestação de serviços aos usuários, também existiram divergências de informações em relação aos serviços especializados, de fato ofertados. Em quase todos os serviços cadastrados $(3,4,5,6$ e 7$)$, as informações não correspondiam à realidade apresentada.

Em boa parte dos serviços analisados, percebeu-se uma alta concentração de pacientes da capital e interior, refletindo assim os vazios assistenciais nas regiões de saúde mais periféricas do Estado. A baixa oferta de fisiatras corroborava para tempo de espera da primeira avaliação física entre três a sete meses, postergando o início da reabilitação dos usuários. Por outro lado, para os pacientes que necessitavam de ostomia (procedimento altamente especializado), o atendimento acontecia em sete dias, no máximo.

Apesar de cadastrado como serviço de dispensação de órteses e prótese, no serviço 2 essa atividade inexistia de forma gratuita, embora prestasse atendimento exclusivo para o SUS. Eram comercializadas órteses e próteses para os usuários que necessitavam, mesmo não sendo evidentes indícios de faturamento dos materiais produzidos nos sistemas de informação.

A informação sobre o quantitativo de pacientes atendidos mensalmente não foi precisa em nenhum dos serviços, pelo desconhecimento dos dados exatos por parte dos gerentes. O tempo de espera para o primeiro atendimento nos serviços variou de uma semana (serviço 6) a sete meses (serviço 2), dependendo do tipo de serviço e/ou necessidade do usuário.

No tocante às atividades realizadas, quando consideradas todas aquelas necessárias a um serviço de reabilitação, observou-se encaminhamento para outros serviços em seis estabelecimentos e atendimento em grupo e cumprimento dos protocolos assistenciais em cinco apenas. Quatro serviços realizavam orientação familiar, capacitação profissional, suporte psicológico para convívio social e familiar e atendimento multiprofissional. Somente três faziam treino para uso de órteses e próteses, ofertavam informações sobre direito das pessoas com deficiência e se articulavam com outros serviços da rede. Apenas dois dispensavam órteses e próteses e faziam acompanhamento pós-alta. Um serviço realizava atendimento domiciliar e nenhum realizava transporte de pacientes.

Em dois serviços, as ações ficavam circunscritas a orientações de prevenção de incapacidade para os usuários, uma vez que não havia intervenção, em decorrência da precária infraestrutura.

\section{DISCUSSÃO}

Esse estudo revelou insuficiência dos serviços ambulatoriais de reabilitação física do SUS, em Salvador-Ba, para atender à população da capital e do interior do Estado. Essa insuficiência coincidiu com outros achados em sistemas municipais de saúde brasileiros e no mundo.

Na América Latina e no Caribe, programas públicos de reabilitação para pessoas com incapacidades foram incorporados na área da saúde, mas não são considerados prioritários. Os poucos serviços existentes apresentam baixa qualidade e os objetivos da reabilitação raramente são cumpridos, principalmente por dificuldade de financiamento (OPAS, 1996), o que apresenta semelhança com achados deste estudo, dada a não priorização dos serviços de reabilitação durante a alocação de recursos.

Esse estudo evidenciou desequilíbrio entre a demanda de usuários com deficiência e a oferta de serviços de reabilitação física. Tal problema também foi evidenciado em sistemas municipais de saúde em cinco capitais brasileiras, revelando contradições na garantia do direito ao atendimento integral em reabilitação (DESLANDES et al., 2006).

A falta de priorização na assistência em reabilitação pelos entes municipais parece um problema recorrente em capitais brasileiras. No do Rio de Janeiro, por exemplo, há apenas um estabelecimento especializado na área e em Manaus, o poder público municipal não desempenha atividades no eixo da reabilitação, concentrando a responsabilidade sobre o nível estadual (DESLANDES et al., 2006).

Verificou-se uma iniciativa pontual da Secretaria Municipal de Saúde (SMS) para credenciamento de serviços de reabilitação física, mental, visual e múltiplas deficiências, a fim de atender a demanda de Salvador. Ao verificar a relação dos 16 serviços habilitados para esse edital, apenas um deles possuía cadastro de serviços de reabilitação física. Os demais serviços habilitados tratavam-se de casa de repouso, centros de reabilitação mental e visual, clínicas de fisiatria, de ginecologia e obstetrícia. Essa importante incoerência aponta para grave inconsistência administrativa que descortina duas questões centrais: a primeira, relativa a interpretações equivocadas da gestão da SMS sobre a natureza dos serviços de reabilitação física; e a segunda, referente aos critérios de credenciamento das unidades, dado distanciamento entre as atividades prestadas pela maioria dos serviços em relação à alta demanda por reabilitação física evidente na capital. 
Embora a assistência de fisioterapia tenha o objetivo de prevenir e tratar os distúrbios cinéticos funcionais intercorrentes em órgãos e sistemas do corpo humano e a fisiatria seja uma especialidade que também aborde as mais variadas alterações de função nos pacientes, a reabilitação física é um processo muito mais amplo e envolve o trabalho de vários profissionais de saúde (UNION EUROPÉENNE DES MÉDECINS SPÉCIALISTES, 2009). Tais profissionais devem estar alocados em serviços especializados com estrutura própria, para estabelecimento de plano terapêutico, a partir das necessidades particulares de cada indivíduo, de acordo com os impactos da deficiência sobre sua funcionalidade (OMS, 2011).

Em virtude da quase totalidade dos serviços habilitados pela SMS não corresponderem a serviços especializados que comportem estrutura adequação à reabilitação física, surge a indagação em relação ao tipo de assistência que a população com deficiência física está acessando, e se esta tem sido suficiente para devolver aos usuários, as condições necessárias para a sua reinserção social e profissional.

Além da ausência de serviços de reabilitação física municipais, apenas um dentre os investigados estavam sob gestão municipal, porém com esfera administrativa privada, revelando que a assistência em reabilitação física na cidade é condicionada pela lógica de contratação de serviços pelo SUS e de grande participação do setor privado.

Embora o PMS 2010-2013 aponte a necessidade da ampliação da rede municipal de atenção especializada própria, o que se evidencia é a reprodução de práticas de gestão de serviços semelhantes às da década 70 , com grande expansão da oferta e da participação do setor privado na prestação de serviços de saúde, financiados pelo Estado (BRASIL, 2011). Uma vez que a estratégia adotada pela gestão municipal é a celebração de contratos privados em detrimento de uma organização de rede própria, convém salientar possibilidades de baixa capacidade estatal regulatória para monitorar e avaliar os serviços prestados, o que parece caracterizar uma redução de autonomia do SUS diante do "próprio SUS".

A inexistência do contrato de gestão e metas em quase todos os serviços fere os princípios do direito administrativo (LIMA, 2012) e evidencia a ausência de mecanismos efetivos de monitoramento e avaliação pela gestão. Esse fato ratifica o quão frágil é a legitimidade na relação entre as partes e a garantia da oferta e atendimento às necessidades em reabilitação física, uma vez que se tem um setor público subfinanciado e um sistema privado sub-regulado.

Muitas foram as divergências encontradas entre as informações contidas no CNES e a realidade dos serviços selecionados neste estudo, o que demonstrou fragilidade do cadastro nas suas funções de propiciar o conhecimento da rede assistencial em saúde e de cumprimento de todas as etapas citadas do processo de cadastramento das unidades (BRASIL, 2011), principalmente no que diz respeito à verificação in loco pelo gestor. Essa situação aponta não somente a fragilidade do sistema de informação e seu uso para apoiar a gestão dos serviços, como também revela um descompasso significativo entre o negociado e o efetivamente prestado.

Os problemas de estrutura física aqui evidenciados ratificam a insuficiência histórica de infraestrutura de serviços públicos de saúde na cidade de Salvador (TEIXEIRA, 2010) e percebe-se a repetição dessa situação também outras capitais como Rio de Janeiro, Curitiba e Brasília (DESLANDES et al., 2006).

Quando se consideram problemas infraestruturais de serviços de saúde brasileiros, cabe salientar as desigualdades na sua distribuição espacial em termos de instalações, equipamentos e do pessoal de saúde, com expressivos vazios assistenciais, sobretudo nas regiões Norte e Nordeste. Estes problemas enfrentam obstáculos que também são políticos e que envolvem relações de poder entre os entes federativos (LIMA, 2012), na tomada de decisão em políticas públicas. Isso justifica a necessidade de compreensões mais aprofundadas dessas relações e dos interesses que permeiam as decisões que envolvem a assistência em reabilitação.

Apesar dos problemas de infraestrutura nos serviços de atenção especializada estarem elencados nos panos de saúde estadual e municipal, a manutenção desse quadro nos serviços estudados revelou contradições entre o que está posto como projeto e o que, de fato, consegue-se implementar.

Não existiu nos serviços investigados regularidade quanto aos parâmetros de funcionamento obedecidos e, dos três serviços de reabilitação física identificados, somente um referiu ter todas as suas atividades norteadas por documentos normativos do Ministério da Saúde.

O tempo de espera para o primeiro atendimento foi considerado fator problemático, pelo fato de causar impacto nos resultados do tratamento de um paciente que necessita de reabilitação física. Pôde-se evidenciar a existência de lista de espera tanto para o atendimento em reabilitação como para o recebimento de órteses, próteses e meios auxiliares de locomoção, realidade coincidente com o município do Rio de Janeiro (DESLANDES et al., 2006). A falta de acesso à reabilitação ou a demora em iniciar o tratamento pode reduzir o nível de independência e de qualidade de vida dos pacientes, já que a intervenção precoce possibilita a melhoria dos resultados finais ou até mesmo a garantia de uma recuperação total (TURNER-STOKES et al., 2005).

Das quinze atividades a serem realizadas, em apenas um serviço foi evidenciado a realização de quase sua totalidade, com exceção apenas para o transporte de pacientes, que também não foi atividade realizada por nenhum outro serviço e é considerado um problema grave nos serviços de reabilitação de Recife, Rio de Janeiro e Curitiba (CAVALCANTI, 2009). A falta de transporte para os pacientes é apontada como um entrave no tratamento de pacientes sequelados em diferentes locais do país (LIMA et al., 2009). 
A realização de atendimento em grupo foi evidenciada em menos da metade dos serviços, o que coincidiu com os achados para os serviços de reabilitação de Pernambuco (LIMA et al., 2009). A convivência em grupo composto por pessoas com problemas semelhantes proporciona uma experiência que pode desenvolver um clima de valor terapêutico, ajudando os participantes a quebrarem barreiras criadas por sentimentos de solidão e isolamento, especialmente pela possibilidade de feedback e sugestões construtivas de outras pessoas que vivenciam os mesmos problemas (MUNARI; RODRIGUES, 1997).

No que se refere à orientação familiar, percebeu-se a sua realização em apenas um terço dos serviços investigados. Isso fragmenta a participação da família enquanto parte do processo de reabilitação, uma vez que e desempenha papel fundamental seja no processo terapêutico, seja no prognóstico dos usuários (GIANNOTTI-HALLAGE, 1981).

Menos da metade dos serviços tinha implementado suporte psicológico para convívio social e familiar, como componente da reabilitação. A maioria dos serviços dos municípios pernambucanos também não realizava essa atividade (GIANNOTTI- HALLAGE, 1981). A presença da doença física, em geral, constitui fonte de tensão emocional e tende a perturbar o equilíbrio psíquico do paciente, o que faz com que o planejamento para a reabilitação psicológica do paciente com deficiência física tenha importante valor.

A dispensação de órteses e próteses foi evidenciada em apenas dois serviços. E essa situação é agravada pelo fato desses serviços absorverem toda demanda dos usuários de todas as regiões do Estado da Bahia. Trata-se de uma realidade coincidente com a situação dos serviços de reabilitação de Pernambuco (LIMA et al., 2009), onde todos os pacientes são encaminhados para a capital.

A quase ausência de atendimento domiciliar em reabilitação, somada à ausência de transporte sanitário dos serviços para os pacientes que necessitam de tratamento, negligenciam o acesso para aqueles que não possuem condição de deslocamento para os serviços especializados na capital. A atenção domiciliar potencializa o resgate dos princípios doutrinários do SUS, se assumida como prática centrada na pessoa enquanto sujeito do seu processo de saúde-doença-cuidado. No caso da reabilitação pode trazer benefícios ao usuário e sua família, pela possibilidade de ser realizada de forma segura e eficaz no seu ambiente cotidiano, permitindo uma melhor avaliação de suas reais necessidades (COELHO et al., 2004).

O cumprimento de protocolos assistenciais foi uma atividade incipiente nos serviços do estudo e também naqueles analisados por CAVALCANTI (2009). Protocolos assistenciais são considerados formas estruturadas de suporte do manejo clínico que incluem a definição de objetivos terapêuticos e uma sequência temporal de cuidados e estratégias diagnósticas e terapêuticas definidas (POLANCZYK, 1998).

As unidades não realizavam a educação permanente dos profissionais. Paim (1993) ressalta que essas ações se constituem como fundamentais, quando se considera a formação de pessoal para a área da saúde coletiva, uma vez que eles são, simultaneamente, os agentes das práticas de saúde enquanto um "técnico de necessidades sociais de saúde" e um "gerente de produção de serviços" (grifos são do autor).

Quase metade dos serviços não desenvolvia atenção multiprofissional. No estudo de Deslandes et al. (2006), também foi verificada a ausência de atendimento multiprofissional em muitos dos serviços de reabilitação investigados no país e nos municípios de Pernambuco (LIMA et al., 2009).

Nenhum dos serviços de reabilitação física investigados realizava referência e contrarreferência. Em Manaus, de maneira semelhante foi identificada fragilidade do referenciamento da rede para continuidade dos atendimentos e, em Brasília, os problemas nos fluxos de referência e contrarrefêrencia dificultam a chegada do paciente ao serviço de reabilitação (DESLANDES et al., 2006).

Acompanhamento pós-alta e oferta de informações sobre os direitos das pessoas com deficiência foram atividades realizadas em poucos serviços, dois e três respectivamente, e não foram encontrados dados de serviços de outras regiões para comparação em relação a estes aspectos.

Houve dificuldade em manusear dados dos serviços de reabilitação física inseridos em unidades hospitalares, devido ao fato do cadastro organizar apenas os dados gerais dos estabelecimentos, não trazendo a separação das informações por serviço, o que tornou problemática a análise das categorias selecionadas.

A impossibilidade de acesso a um dos gerentes selecionados, a recusa de três serviços em participar do estudo, a pouca receptividade na SMS e o pouco tempo dispendido para as entrevistas constituíram as principais dificuldades metodológicas desse estudo.

A dificuldade de acesso aos gerentes de quatro serviços corroborou para que as evidências ficassem limitadas aos dados do CNES, das páginas eletrônicas dos serviços (quando existentes) e das informações obtidas em entrevista realizada no nível central da gestão estadual. Características da prestação de serviços e problemas que seriam melhor explicitadas pelo cotejamento dos excertos de diferentes atores com os dados secundários, ficaram limitados a esses últimos.

A utilização de dados secundários do CNES também trouxe à tona uma série de divergências entre as informações fornecidas e a situação encontrada, o que constituiu um importante dificultador na realização deste diagnóstico, dada incompatibilidade cadastral com realidade dos serviços. Por outro lado, mostrou-se um potencial revelador de fragilidades cadastrais de serviços de saúde.

\section{CONCLUSÃO}

Os resultados deste momento explicativo alertaram para a importância da formulação de propostas rumo à transformação da situação da assistência em reabilitação física na cidade de Salvador-Ba, que envolva a articulação 
das esferas administrativas de governo na produção/ tradução dos documentos que se destinam à assistência em reabilitação física e implantação das ações planejadas. O objetivo está pautado em fazer valer o esforço legal dos instrumentos normativos; o planejamento e a organização da rede de serviços de reabilitação física com base nas necessidades epidemiológicas da população, a fim de garantir assistência efetiva ao usuário e diminuir o tempo de espera pelo atendimento; o incremento na infraestrutura dos serviços, com disponibilidade de equipe multiprofissional, tecnologia e instalações apropriadas para a realização de todas as atividades necessárias ao processo de reabilitação; o desmame da prática da compra de serviços de reabilitação pelo setor público e o fortalecimento da construção de uma rede própria, que assegure maior autonomia do sistema na organização da assistência em reabilitação.

Faz-se necessário outros estudos, suplantando as limitações encontradas nesta investigação, para que seja possível a elaboração de um diagnóstico mais completo, assim como estudos avaliativos que abarquem análises políticas e estratégicas envolvendo a assistência em reabilitação em saúde, que aprofundem as arenas e os atores nos processos decisórios, desde a sua formulação à implementação.

\section{REFERÊNCIAS}

ALMEIDA, M. C.; CAMPOS, G. W. S. Políticas e modelos assistenciais em saúde e reabilitação de pessoas portadoras de deficiência no Brasil: análise de proposições desenvolvidas nas últimas duas décadas. Rev. Chil. Ter. Ocup., Santiago, v. 13, n. 3, p. 118-126, 2002.

BONARDI, G. et al. Incapacidade funcional e idosos: bum desafio para os profissionais de saúde. Sci Med., Porto Alegre, v. 17, n. 3, p. 138144, 2007.

BRASIL. Ministério da Saúde. Regulação em saúde: coleção para entender a gestão do SUS. Brasília: Ministério da Saúde, 2011. V.10.

BRASIL. Ministério da Saúde. Portaria MS/GM no 1.060, de 5 de junho de 2002. Aprova a Política Nacional da Pessoa Portadora de Deficiência. Brasília: Ministério da Saúde, jun. 2002.

BRASIL. Ministério da Saúde. Portaria MS/GM no 4279 de 30 de dezembro de 2010. Estabelece diretrizes para a organização da Rede de Atenção à Saúde, no âmbito do SUS. Brasília: Ministério da Saúde, jun. 2010.

CAVALCANTI, M. L. T. A análise da implantação da Política Nacional de Redução de Mortalidade por Acidentes e Violências ressalta os problemas do SUS. Ciênc. Saúde Colet., Rio de Janeiro, v. 14, n. 5, p. 1650-1651, 2009.

COELHO, S. C. et al. Assistência domiciliar: a experiência de um hospital privado do interior paulista. Rev. Latinoam. Enfermagem, Ribeirão Preto, v. 12, n. 5, p. 721-726, 2004.

DESLANDES, S. F. et al. Caracterização diagnóstica dos serviços que atendem vítimas de acidentes e violências em cinco capitais brasileiras. Ciênc. Saúde Colet., Rio de Janeiro, v. 11, n. 1, p. 279-1290, 2006.
GIANNOTTI-HALLAGE, A. Reabilitação psicológica de pacientes com sequelas da encefalite por arbovírus. Rev. Saúde pública, São Paulo, v. 15, p. 478-84, 1981.

KIELMANN, S.; SILVEIRA, L.; SOUZA, L. As redes de atenção para pessoas com deficiência na Bahia: construindo políticas públicas. In: Políticas Públicas e Pessoa com Deficiência: direitos humanos, família e saúde. Salvador: EDUFBA. 2011. p. 288.

LIMA, M. L. C. de. et al. Análise diagnóstica dos serviços de reabilitação que assistem vítimas de acidentes e violências em Recife. Ciênc. Saúde Colet., Rio de Janeiro, v. 14, n. 5, p. 1817-1824, 2009.

LIMA, M. L. C. Avaliação da Política Nacional de morbimortalidade por acidentes e violência, nos municípios de Pernambuco com mais de 1000.000 habitantes. Recife: Editora Universitária da UFPE, 2012. 202 p.

MUNARI, D. B.; RODRIGUES, A. R. F. Enfermagem e grupos. Goiânia: AB Editora; 1997.

OMS. Organização Mundial da Saúde. Relatório Mundial sobre a deficiência. Secretaria de Estado dos Direitos da Pessoa com Deficiência de São Paulo. 2011.

OPAS. Organización Panamericana de Saúde. La rehabilitación por discapacidad em América Latina y el Caribe. Bol. Oficina Sanit. Panam., Washington, v. 120, n. 4, p. 358-361, 1996.

PAIM, J. S. Marco de referência para um programa de educação continuada em Saúde Coletiva. Rev. Bras. Educ. Méd., Rio de Janeiro, v. 17, p. 7-13, 1993.

POLANCZYK, C. A. et al. Protocolos Assistenciais como Estratégia de Adesão às Melhores Práticas Clínicas e Otimização de Recursos. Ministério da Educação. Hospital de Clínicas de Porto Alegre (HCPA). Relato de experiência. 1998.

REICHENNHEIM, M. E. et al. Violência e lesões no Brasil: efeitos, avanços alcançados e desafios futuros. Lancet, London, v. 6736, n. 11, p. 60433-60439, 2011.

RIBEIRO, C. T. et al. O sistema público de saúde e as ações de reabilitação no Brasil. Rev. Panam. Salud Publica, Washington, v. 28, n. 1, p. 43-48, 2010.

SCHMIDT, M. I. et al. Chronic non-comminicable diseases in Brazil: burden and current challenges. Lancet, London, v. 377, n. 9781, p. 1949-1961, 2011.

SUS: o desafio da saúde para todos. Revista CONSENSUS, Conselho Nacional de Secretários de Saúde. Ano 1, n. 1, jul./set. 2011

TEIXEIRA, C. Planejamento em Saúde: conceitos, métodos e experiências. Salvador: EDUFBA, 2010. p. 161.

TESTA, M. Pensamento estratégico e lógica de programação: o caso da saúde. Hucitec: São Paulo, ABRASCO: Rio de Janeiro. 1995.

TURNER-STOKES, L. et al. Multi-disciplinary rehabilitation for acquired brain injury in adults of working age. Cochrane Database Syst. Rev., Oxford, n. 3, 2005.

UEMS. Union Européenne Des Médecins Spécialistes. Livro Branco de Medicina Física e de Reabilitação na Europa. Secção de Medicina Física e de Reabilitação da Union Européenne des Médecins Spécialistes (UEMS). 2009. 